\title{
(Pseudo-) kyste salivaire sur les glandes de Blandin-Nuhn
}

\author{
Fanny Elsig ${ }^{1, *}$, Fabien Hauser ${ }^{1,2}$, Tommaso Lombardi ${ }^{3}$, Jacky Samson ${ }^{1,2}$ \\ 1 Centre médico-dentaire de Balexert, Avenue Louis-Casaï 27, 1209 Genève, Suisse \\ 2 Division de Stomatologie et Chirurgie orale, Faculté de Médecine, Genève, Suisse \\ 3 Laboratoire d'Histopathologie buccale, Faculté de Médecine, Genève, Suisse \\ *fannyelsig@hotmail.com
}

(Reçu le 20 mai 2011, accepté le 20 juin 2011)

Mots clés :

pseudo-kyste salivaire / langue /

Blandin-Nuhn

Key words:

mucocele / tongue / Blandin-Nuhn

\begin{abstract}
Résumé - Comme la plupart des autres glandes salivaires accessoires, les glandes de Blandin-Nuhn donnent parfois naissance à des pseudo-kystes. Ils siégent sur la partie antérieure de la face ventrale de la langue et s'observent surtout chez l'enfant et l'adulte jeune. Les glandes, regroupées mais non encapsulées, sont situées assez profondément dans les muscles de la langue et une récidive peut survenir si l'exérése n'intéresse pas la totalité des glandes.
\end{abstract}

\begin{abstract}
Mucocele of the gland of Blandin-Nuhn. As most other minor salivary glands, the glands of Blandin-Nuhn may occasionally raise to mucous extravasation mucoceles located on the anterior ventral surface of the tip of the tongue. Most patients were children or young adults. The glands are grouped but not encapsulated, deeply embedded within the muscles. An incomplete excision that not comprise all the affected gland is often followed by a recurrence.
\end{abstract}

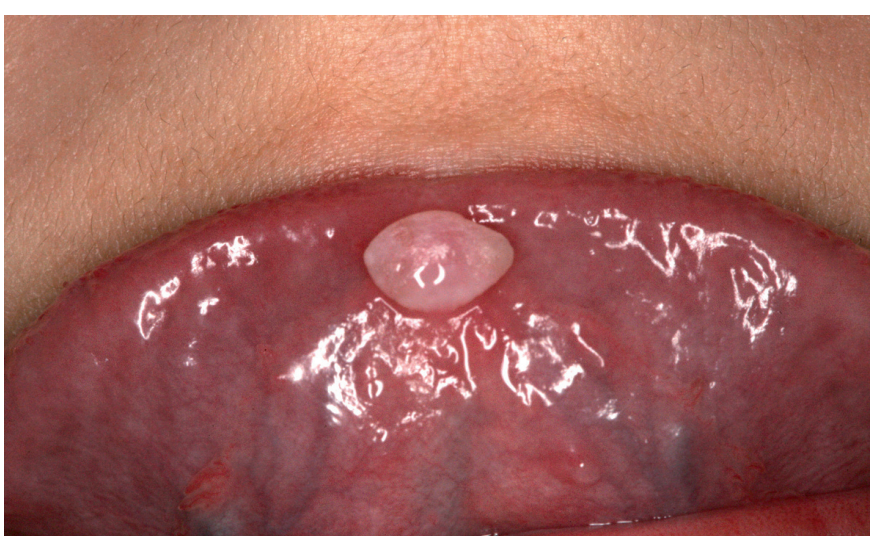

Fig. 1. Tumeur pseudo-pédiculée, translucide, ovalaire, mesurant $6 \times 4 \mathrm{~mm}$, sur la face ventrale de la pointe de la langue.

Fig. 1. Translucent, oval, pseudo-pediculated tumor, of $6 \times 4 \mathrm{~mm}$, located on the middle of the ventral tip of the tongue.

Une jeune fille de 15 ans est adressée par son orthodontiste pour une lésion sur la face ventrale de la pointe de la langue évoluant depuis 2 mois. La patiente décrit une "boule » indolore dont la taille varie constamment. A l'anamèse, on ne retrouve pas d'antécédents particuliers, ni la notion de traumatisme dans cette région.
L'examen initial montre qu'il s'agit d'une tumeur pseudo-pédiculée, translucide, rénitente, ovalaire, mesurant $6 \times 4 \mathrm{~mm}$, siégeant dans la région médiane de la face ventrale de la pointe de la langue. L'aspect clinique (Fig.1) fait évoquer un pseudo-kyste salivaire développé à partir des glandes de Bandin-Nuhn. Lors de l'exérése programmée 15 jours plus tard, la lésion avait presque disparu.

L'exérése a été réalisée sous anesthésie locale avec dissection de la partie profonde des glandes de BlandinNuhn; elles occupaient la moitié de l'épaisseur de la langue et la pièce d'exérése mesurait $10 \times 5 \times 7 \mathrm{~mm}$. Il n'y a pas eu de récidive. L'examen histopathologique a montré un reliquat du pseudo-kyste représenté par un petit épanchement de salive entouré par un tissu de granulation inflammatoire. Les glandes salivaires au voisinage étaient le siége de lésions de sialadénite chronique avec un infiltrat lymphoplasmocytaire diffus prédominant dans la zone péricanaliculaire, et une fibrose inter-lobulaire et inter-acineuse avec ectasie de certains canaux excréteurs. 


\section{Commentaires}

Les glandes salivaires accessoires donnent assez fréquemment naissance à des kystes, soit par extravasation (pseudokystes), les plus fréquents, soit par rétention (vrais kystes), assez rares. Elles sont très abondantes mais leur densité et leur répartition varient selon les régions anatomiques. Celles de la langue présentent quelques particularités : elles sont séreuses, muqueuses ou séro-muqueuses et leur distribution est limitée à certaines régions de la langue. On distingue trois groupes : les glandes à prédominance séreuse de Von Ebner siégeant dans la région des papilles caliciformes et des papilles foliées, les glandes muqueuses de Weber sur les bords de la langue et l'amygdale linguale, et les glandes séromuqueuses de Blandin-Nuhn (nom de deux anatomistes, l'un français Philippe Frédéric Blandin 1798-1849, l'autre allemand Anton Nuhn 1814-1891) dans la partie ventrale médiane de la pointe de la langue $(3,4)$. Les glandes de Blandin-Nuhn forment un groupe compact mais elles ne sont pas encapsulées, et elles sont assez profondément enchassées dans les muscles de la langue. Elles se drainent par 5 ou 7 canaux dont l'orifice se trouve en avant ou dans la région de l'extrémité antérieure du frein de la langue $(1-3,5)$. Ce sont les seules glandes salivaires linguales qui semblent pouvoir donner naissance à une pathologie (kystes ou tumeurs) mais celle-ci reste relativement rare ; les kystes s'observent chez des enfants et des adultes jeunes, les tumeurs chez des sujets plus âgés. Elles touchent indifféremment les deux sexes sauf pour Jinbu et al. qui retrouvent une nette prédominance féminine ( $4 \mathrm{~F}$ pour $1 \mathrm{M}$ ) dans leur série de 26 cas (4).

Les kystes développés sur les glandes de Blandin-Nuhn se présentent comme une tumeur médiane ou para-médiane, plus rarement latérale, siégeant sur la face ventrale de la pointe de la langue. Cette tumeur, séssile ou pédiculée, est indolore, le plus souvent de couleur rouge, quelquefois translucide et/ou bleutée, de consitance rénitente ou élastique; elle mesure en moyenne $6 \mathrm{~mm}$ de diamétre (4). Les lésions antérieures sont le plus souvent (pseudo-)pédiculées et médianes, les lésions postérieures sessiles et latérales.

L'aspect clinique peut faire évoquer une lésion vasculaire, un granulome pyogènique, un polype, un papillome ou une autre tumeur (2). En général, il n'est pas nécessaire de réaliser une ponction pour confirmer le diagnostic.

L'exérése doit comporter une dissection soigneuse de la totalité des glandes salivaires si l'on veut éviter toute récidive. La marsupialisation aboutit à un échec car les berges se collabent spontanément $(1,3)$. L'injection de produit d'empreinte, préconisée par certains auteurs, pour stabiliser la lésion et faciliter sa dissection, peut aboutir à la diffusion du produit dans les tissus du voisinage (3).

L'examen histopathologique montre qu'il s'agit d'un pseudokyste (cavité kystique sans bordure épithéliale, contenant de la salive, entourée par une réaction inflammatoire) associé à une sialadénite chronique intéressant les glandes du voisinage. Cet aspect est caractéristique d'une extravasation salivaire, probablement secondaire à un traumatisme dont la notion est rarement retrouvée à l'interrogatoire.

\section{Conflits d'intérêt : aucun}

\section{Références}

1. Adachi P, Pires Soubhia AM, Horikawa FK, Shinohara EH. Mucocele of the glands of Blandin-Nuhn : clinical, pathological and therapeutical aspects. Oral Maxillofac Surg 2011;15:11-3.

2. De Camargo P, Bönecker M, Furuse C, Thomaz LA, Gonçalves Teixeira R, Cavalcanti de Araujo V. Mucocele of the gland of Blandin-Nuhn : histological and clinical findings. Clin Oral Invest 2009;13:351-3.

3. Ellis E 3rd, Scott R, Upton LG. An unusual complication after excision of a recurrent mucocele of the anterior lingual gland. Oral Surg Oral Med Oral Pathol 1983;56:467-71.

4. Jinbu Y, Kusama M, Itoh H, Matsumoto K, Wang J, Noguchi T. Mucocele of the glands of Blandin-Nuhn : clinical and histopathologic analysis of 26 cases. Oral Surg Oral Med Oral Pathol Oral Radiol Endod 2003;95:467-70.

5. Sugerman PB, Savage NW, Young WG. Mucocele of the anterior lingual salivary glands (glands of Blandin and Nuhn) : report of 5 cases. Oral Surg Oral Med Oral Pathol Oral Radiol Endod 2000;90:478-82. 